\title{
Insulin resistance and development of diabetes mellitus associated with megestrol acetate therapy
}

\author{
Prasoon Jain, Luigi S Girardi, Lawrence Sherman, Michael Berelowicz, Lawrence G Smith
}

\begin{abstract}
Summary
We describe a case of diabetes mellitus induced by megestrol acetate in a patient with the acquired immunodeficiency syndrome. Metabolic studies including an arginine infusion test excluded an insulinopenic state and suggested insulin resistance as the underlying mechanism for hyperglycaemia. Withdrawal of megestrol acetate resulted in rapid correction of all metabolic abnormalities and eliminated the need for exogenous insulin therapy.
\end{abstract}

Keywords: diabetes mellitus, megestrol acetate, AIDS, insulin resistance

Megestrol acetate, a synthetic orally active progestational agent has recently been used for the treatment of weight loss and cachexia associated with cancer and the acquired immunodeficiency syndrome (AIDS). It is thought that megestrol acetate promotes weight gain by stimulating appetite; however, the exact mechanism remains unknown. In general, the drug is well-tolerated with few potential adverse effects including peripheral oedema, impotence, menstrual irregularities, deep vein thrombosis and hyperpnea. Recently, we encountered a case of diabetes mellitus induced by megestrol acetate in a patient with AIDS.

\section{Case report}

A 36-year-old homosexual man with AIDS was admitted to hospital with a diagnosis of hyperosmolar nonketotic syndrome. One year prior to admission, the patient had suffered from Pneumocystis carinii pneumonia, successfully treated with intravenous pentamidine. Since then he had been maintained on dapsone for secondary prophylaxis without recurrence of the $P$ carinii pneumonia. Antiretroviral therapy consisted of dideoxyinosine which was discontinued six months prior to admission because of persistent but asymptomatic elevation of pancreatic enzymes.

Physical examination revealed dehydration, mild confusion and slurring of speech. No organomegaly or signs of localised infection were noted. His height, weight and body mass index were $172 \mathrm{~cm}, 66 \mathrm{~kg}$ and $22.3 \mathrm{~kg} / \mathrm{m}^{2}$, respectively.

Laboratory data included the following: blood glucose $51 \mathrm{mmol} / \mathrm{l}$, sodium $129 \mathrm{mmol} / \mathrm{l}$, potassium $5.4 \mathrm{mmol} / \mathrm{l}$, bicarbonate $17 \mathrm{mmol} / \mathrm{l}$, triglyceride $\quad 11.5 \mathrm{mmol} / \mathrm{l}$, cholesterol 5.7 $\mathrm{mmol} / \mathrm{l}$, amylase $111 \mathrm{U} / 1$ (normal $7-59 \mathrm{U} / \mathrm{l}$ ), and lipase $140 \mathrm{U} / 1$ (normal 0-88 U/l). Arterial blood gas: $\mathrm{pH}$ 7.45, $\mathrm{pCO}_{2} 20 \mathrm{mmHg}, \mathrm{pO}_{2}$ $127 \mathrm{mmHg}$ on room air. Serum osmolality was $322 \mathrm{mOsm} / \mathrm{kg}$. Serum and urinary ketone were negative. Chest X-ray, electrocardiogram and computed tomography (CT) scan of the abdomen were normal.

Intravenous fluids and insulin were administered with resultant rapid improvement in condition. Over the next several days, the patient required up to 75 units of insulin in 24 hours for glycaemic control.

Careful review of his records revealed that the patient was started on megestrol acetate, $240 \mathrm{mg} /$ day, three months prior to admission for progressive anorexia and weight loss. One week after starting megestrol acetate, hyperglycaemia was noted for the first time on random blood glucose testing. An oral glucose tolerance test six weeks later was abnormal (table 1). Megestrol acetate induced diabetes mellitus was therefore strongly suspected and further metabolic studies were pursued. A fasting Cpeptide level was $1.09 \mathrm{mmol} / 1$ (normal $0.1-$ $1.22 \mathrm{mmol} / \mathrm{l})$. Anti-insulin antibodies were negative. Pancreatic islet cell function was studied after intravenous infusion of arginine $(0.5 \mathrm{~g} / \mathrm{kg}$ over $30 \mathrm{~min})$. C-Peptide, insulin and glucagon responses were normal (table 2). These results demonstrated that islet cell function was intact. Megestrol acetate was subsequently discontinued, and insulin requirements rapidly declined, stopping altogether after one week. The patient remained euglycaemic and a repeat oral glucose tolerance test after two weeks was within normal limits (table 1). A repeat serum triglyceride at this time was $2.86 \mathrm{mmol} / \mathrm{l}$, also consistent with the correction of insulin resistance.

Table 1 Oral glucose tolerance test after $75 \mathrm{~g}$ of glucose performed six weeks after starting megestrol acetate and two weeks after withdrawing the drug

\begin{tabular}{ccl}
\hline & \multicolumn{2}{l}{ Blood glucose (mmolll) } \\
\cline { 2 - 3 } Time (min) & $\begin{array}{l}\text { Six weeks after } \\
\text { starting }\end{array}$ & $\begin{array}{l}\text { Two weeks after } \\
\text { stopping }\end{array}$ \\
\hline 0 & 5.0 & 4.3 \\
30 & 11.5 & 6.9 \\
60 & 11.5 & 8.2 \\
120 & 12.0 & 7.6 \\
\hline
\end{tabular}


Table 2 C-Peptide, insulin, glucagon response after intravenous infusion of arginine $(0.5 \mathrm{~g} / \mathrm{kg}$ over $30 \mathrm{~min}$ )

\begin{tabular}{clll}
\hline Time (min) & C peptide $^{\star}$ & Insulin $^{\star *}$ & Glucagon \\
\hline 0 & 1.0 & 136 & 210 \\
30 & 2.0 & 230 & 530 \\
60 & 2.4 & 244 & 530 \\
90 & 2.7 & 179 & 210 \\
120 & 3.5 & 244 & 260
\end{tabular}

*Normal 0.1-1.22 mmol//; ${ }^{\star}$ normal 35-145 pmol/1; tnormal 50-100 ng/1

\section{Discussion}

Many observations suggest that megestrol acetate precipitated diabetes mellitus in this patient. A strong temporal relation was noted between initiation of megestrol acetate therapy and the onset of hyperglycaemia and an abnormal oral glucose tolerance test. While receiving megestrol acetate, the patient required large doses of insulin to maintain adequate glycaemic control after correction of his hyperglycaemic nonketotic state. Rapid decrease in insulin needs, subsequent maintenance of euglycaemia without insulin, and normalisation of oral glucose tolerance test upon discontinuation of the drug clearly indicate a primary role of megestrol acetate.

Various endocrine abnormalities have been observed in patients with AIDS (box 1).$^{1-4} \mathrm{We}$ strongly suspect megestrol-induced insulin resistance to be the cause of diabetes mellitus in our patient. A high-normal fasting Cpeptide level during the hyperglycaemic state and an adequate insulin and glucagon secretory response to arginine infusion ruled out the possibility of drug-induced islet cell damage resulting from prior pentamidine therapy. Insulin resistance is also suggested by the presence of gross hypertriglyceridaemia when the patient was admitted to hospital, with rapid normalisation of triglyceride levels after megestrol acetate therapy was withdrawn.

The exact metabolic alterations leading to weight gain with megestrol acetate therapy are unknown. Loprinzi et $a \bar{p}$ performed body composition studies before and two months after starting megestrol acetate in 12 patients with advanced cancer and showed that in creases in fat account for most of the weight gained during therapy. In an in vitro model, megestrol acetate promoted differentiation of 3T3-L1 Swiss mouse embryo fibroblasts to adipocytes. $^{6}$ The effects of megestrol acetate, a progestational agent, on glucose metabolism are also poorly understood. Henry et $a{ }^{\beta}$ postulated glucocorticoid-like activity of megestrol as the possible cause of insulin resistance in their patient. Inhibitory effects of progesterone on phosphorylation of glucose into glucose-6-phosphate ${ }^{7}$ and inhibition of insulin-stimulated transmembrane glucose

\section{Endocrine abnormalities and AIDS}

- acute pancreatitis: complication of dideoxyinosine, pentamidine, and trimethoprin - sulfamethoxazole therapy

- hypoglycaemia: complication of intravenous pentamidine therapy (beta cell toxicity)

- diabetes mellitus: complication of intravenous pentamine and megestrol

Box 1

\section{Summary/learning points}

- in appropriate clinical settings, megestro acetate-induced insulin resistance should be included in the differential diagnosis of hyperglycaemia in patients with AIDS

- withdrawal of megestrol acetate may result in rapid correction of all metabolic abnormalities

Box 2

transport, ${ }^{8}$ noted in animal studies, may partly explain a progesterone-induced insulin resistant state. In humans, mild and subclinical alterations in glucose tolerance and presumably insulin resistance has been noted with the use of continuous subdermal levonorgestrel. ${ }^{9}$ Interestingly, no adverse effect on blood glucose $^{10}$ or lipid profile ${ }^{5}$ have been noted in cancer patients treated with high-dose megestrol acetate. It is commonly accepted that patients with AIDS show a higher incidence of adverse drug reactions. For this reason, patients with AIDS may be particularly at risk of developing severe glucose disturbances while taking megestrol acetate.

We realise that some patients presenting with hyperosmolar nonketotic coma show rapid improvement in metabolic profile after receiving intensive supportive care with intravenous fluid and insulin. This is especially true when an underlying precipitating cause is promptly identified and corrected. Some of these individuals may not require long-term insulin therapy to control blood glucose. However, a majority of these patients continue to show some evidence of metabolic derangement on formal glucose tolerance testing. Although we cannot exclude this possibility with absolute certainty, complete normalization of all metabolic abnormalities after the withdrawal of megestrol acetate argue against this scenario in our case. Therefore, we suggest that blood glucose should be monitored during megestrol acetate therapy in AIDS patients. This is especially important since life-threatening metabolic derangements may occur, as in our patient, which rapidly normalise once the medication is discontinued, obviating the need for insulin therapy. Further studies are needed to define the incidence and mechanism of insulin resistance induced by megestrol acetate in patients with AIDS. 
1 Bouchard PH, Sai P, Reach G, Caubarrere I, Ganeval D, Assan R. Diabetes mellitus following pentamidine-induced hypoglycemia in humans. Diabetes 1982; 31: 40-5.

2 Vendrell J, Nubiola A, Goday A, et al. HIV and the pancreas. Lancet 1987; ii: 1212 .

3 Henry K, Rathgaber S, Sullivan C, McCabe K. Diabetes mellitus induced by megestrol acetate in a patient with AIDS and cachexia. Ann Intern Med 1992; 116: 53-4.

4 Panwalker AP. Hyperglycemia induced by megestrol acetate. Ann Intern Med 1992; 116: 878.

5 Loprinzi CL, Schaid DJ, Dose AM, Burnham NL, Jensen $M D$. Body composition changes in patients who gain weight while receiving megestrol acetate. $\mathcal{F}$ Clin Oncol 1993; 11: $152-4$

6 Hamburger AW, Parnes H, Gordon GB, Shantz LM, O'Donnell KA, Aisner J. Megestrol acetate induced differentiation of 3T3-L1 adipocytes in vitro. Semin Oncol 1988; 15 (suppl 1): 76-7.
7 Sutter-Dub M-Th, Dazey B, Hamden E, Vergnaud M-Th Progesterone and insulin resistance: studies of progesterone Prosterone action on glucose transport, lipogenesis and lipolysis in 455-62.

8 Rushakoff RJ, Kalkhoff RK. Effect of pregnancy and sex steroid administration on skeletal muscle metabolism in the rats. Diabetes 1981; 30: $545-50$

9 Konje JC, Otolorin EO, Ladipo OA. The effect of continuous subdermal levonorgestrel (Norplant) on carbohydrate metabolism. Am f Obstet Gynecol 1992; 166: 15-9. 10 Feliu J, Gonzalez-Baron M, Berrocal A, et al. Usefulness of megestrol acetate in cancer cachexia and anorexia. $A m \mathcal{f}$ Clin Oncol 1992; 15: 436-40.

\title{
Digoxin toxicity presenting as encephalopathy
}

\author{
JR Greenaway, B Abuaisha, MG Bramble
}

\section{Summary \\ We describe two cases of digoxin toxicity presenting with clinical and electroence- phalographic evidence of encephalopathy without other features of digoxin toxicity.}

Keywords: digoxin toxicity, encephalography

Digoxin toxicity often presents with gastrointestinal side-effects such as anorexia, nausea or vomiting. ${ }^{1}$ Other side-effects include cardiac dysrhythmias, visual disturbance and drowsiness. $^{2}$

Case 1

A 68-year-old woman was admitted to hospital with a one-week history of profound somnolence. She had been non-specifically unwell in the preceding month and had fallen twice without any history of head injury. There was no history of anorexia, nausea, vomiting or visual disturbance. Her past medical history consisted of ischaemic heart disease, congestive cardiac failure, atrial fibrillation, cerebrovascular disease and chronic airflow limitation. Medication comprised frusemide $120 \mathrm{mg}$, spironolactone $100 \mathrm{mg}$, prednisolone $5 \mathrm{mg}$, ranitidine $150 \mathrm{mg}$, digoxin $250 \mu \mathrm{g}$, all daily, with isosorbide mononitrate $20 \mathrm{mg}$ and nifedipine SR $10 \mathrm{mg}$ both twice daily.

Clinical examination revealed no focal neu-

Department of

Medicine,

Middlesbrough

General Hospital,

Ayresome Green Lane,

Middlesbrough,

Cleveland TS5 5AZ,

UK

JR Greenaway

B Abuaisha

MG Bramble

Accepted 21 September 1995 rological signs but confirmed extreme drowsiness. Investigations revealed no abnormality of full blood count, urea and electrolytes, liver function tests, blood glucose, thyroid function tests or B12 and folate. Blood cultures and VDRL were negative. Chest X-ray was normal and electrocardiogram showed sinus rhythm with lateral ST depression. Computed tomography (CT) scan of brain revealed no abnormality and lumbar puncture resulted in clear, colourless cerebrospinal fluid with no growth, normal glucose and mildly raised protein at $0.63 \mathrm{~g} / 1$ (normal $<0.4 \mathrm{~g} / \mathrm{l})$. Electroencephalogram (EEG) showed focal right anterior quadrant delta slowing consistent with Herpes encephalitis. However, following a telephone conversation with her general practitioner it transpired that her digoxin dose had been doubled to $250 \mu \mathrm{g}$ daily approximately one month prior to admission. Her digoxin level when checked was $>5 \mu \mathrm{g} / 1$. Digoxin was withheld and the patient gradually woke up. One week later she was back to normal (digoxin level $0.3 \mu \mathrm{g} / \mathrm{l}$ ).

\section{Case 2}

A 66-year-old woman with a previous history of rheumatic valvular heart disease (mitral and aortic valve replacements), atrial fibrillation, chronic congestive cardiac failure and angio-

\begin{tabular}{|l|}
\hline Digoxin: CNS side-effects \\
\hline - lethargy and fatigue \\
- weakness \\
- headache \\
visual disturbances (colour perception \\
problems (including xanthopsia), reduced \\
visual acuity, visual field defects, pain on eye \\
- movement) \\
- pepression \\
- drowsinsis \\
- confusion \\
- delerium \\
- encephalopathy \\
- hallucinations \\
- epileptic seizures \\
- choreiform movements \\
- dysphonia \\
- dysphagia \\
\hline
\end{tabular}

Box 1 